This manuscript has been authored by UT-Battelle, LLC under Contract No. DE-AC05-00OR22725 with the U.S. Department of Energy. The United States Government retains and the publisher, by accepting the article for publication, acknowledges that the United States Government retains a non-exclusive, paid-up, irrevocable, world-wide license to publish or reproduce the published form of this manuscript, or allow others to do so, for United States Government purposes. The Department of Energy will provide public access to these results of federally sponsored research in accordance with the DOE Public Access Plan (http://energy.gov/downloads/doe-public-access-plan). 


\title{
Direct synchrotron x-ray measurements of local strain fields in elastically and plastically bent metallic glasses
}

\author{
Y. Wu, ${ }^{\mathrm{a}, \mathrm{b}, \mathrm{c}}$ A.D. Stoica, ${ }^{\mathrm{d}}$ Y. Ren, ${ }^{\mathrm{e}}$ D. Ma, ${ }^{\mathrm{d}, 1}$ Y.F. Gao, ${ }^{\mathrm{a}, \mathrm{c}, 1}$ H. Bei ${ }^{\mathrm{a}, 1}$ \\ ${ }^{a}$ Materials Science and Technology Division, Oak Ridge National Laboratory, Oak Ridge, TN 37831, USA \\ ${ }^{\mathrm{b}}$ State Key Laboratory for Advanced Metals and Materials, University of Science and Technology Beijing, Beijing, \\ 100083, China \\ ${ }^{c}$ Department of Materials Science and Engineering, University of Tennessee, Knoxville, TN 37996, USA \\ ${ }^{d}$ Chemical and Engineering Materials Division, Spallation Neutron Source, Oak Ridge National Laboratory, Oak \\ Ridge, TN 37831, USA \\ ${ }^{\mathrm{e}}$ Advanced Photon Source, Argonne National Laboratory, Argonne, Illinois 60439, USA
}

\begin{abstract}
In situ high-energy synchrotron X-ray diffraction was conducted on elastically and plastically bent bulk metallic glass (BMG) thin plates, from which distinct local elastic strain fields were mapped spatially. These directly measured residual strain fields can be nicely interpreted by our stress analysis, and also validate a previously proposed indirect residualstress-measurement method by relating nanoindentation hardness to residual stresses. Local shear strain variations on the cross sections of these thin plates were found in the plastically bent BMG, which however cannot be determined from the indirect indentation method. This study has important implications in designing and manipulating internal strain fields in BMGs for the purpose of ductility enhancement.
\end{abstract}

Keywords: A. metallic glasses; B. mechanical properties; F. diffraction/scattering

${ }^{1}$ Corresponding authors: beih@ornl.gov; dongma@ornl.gov; ygao7@utk.edu 


\section{Introduction}

Although bulk metallic glasses (BMGs) have a unique combination of superior mechanical, chemical and physical properties, their room-temperature brittleness has been a long-standing obstacle for structural engineering applications [1-4]. Toughening BMGs is thus

the focus of many investigations over the years [5-9]. Among them, in a manner similar to tempered window glasses, introducing residual stresses onto the surfaces of BMGs has been found to be a unique and effective way in delaying the initiation of shear bands and preventing their transition into micro-cracks [10,11]. Therefore, it is of essential importance to quantitatively determine the residual stress and strain components in order to better understand the relationship between residual stresses and macroscopic mechanical behavior of BMGs. In recent works [12,13], effects of residual stress on yield strength and hardness have been thoroughly studied by nanoindentation experiments. An interesting finding is that the BMG hardness varies dramatically with respect to the tensile residual stress but not to the compressive one, when these residual stresses are applied in the direction orthogonal to the indentation direction. Thus the nanoindentation hardness mapping, which can be conducted easily and efficiently, provides an indirect way to measure residual stresses with a very high spatial resolution.

There are several other approaches for BMG ductility enhancement that rely on geometric constraints and also residual stresses. For example, the introduction of hard and ductile coatings will prevent shear band propagation to the surface, and a further introduction of compressive residual stress will make the above constraining more effective [14]. In BMG composites, the ductile crystalline second-phases will provide geometric obstacles to the shear band propagation, and residual stresses near these second phases control how these shear bands 
are arranged $[15,16]$. A quantitative measurement of the residual strain components will not only validate the above indirect nanoindentation method, but more importantly provide quantitative information on internal strain fields and thus shear band characteristics.

In this work, we have conducted synchrotron x-ray diffraction measurements of local strain fields in elastically and plastically bent BMG thin plates. These directly measured strain fields, which agree well with our stress analysis, not only validate the nanoindentation-based residual-stress measurement method, but also provide the first experimental evidence of the measurement of all strain components inside the BMG specimen.

\section{Experimental details}

BMGs with a nominal composition of $\mathrm{Zr}_{52.5} \mathrm{Al}_{10} \mathrm{Ti}_{5} \mathrm{Cu}_{17.9} \mathrm{Ni}_{14.6}$ (in atomic percent) were prepared by arc melting and subsequent drop casting. Thin plates of 0.6 to $2.5 \mathrm{~mm}$ thick, $3 \mathrm{~mm}$ wide and $15.3 \mathrm{~mm}$ long, were cut from a $7 \mathrm{~mm}$-diameter BMG rod by electrical discharge machining. The elastically bent samples were made by elastic bending of a thin plate, followed by constraining it in a steel ring with an internal diameter of $15 \mathrm{~mm}$ [12]. Regardless of how the bending was made initially, once the sample was placed in the ring and external loading was removed, a pure bending state would be realized. The plastically bent samples were prepared by three-point bending to different curvatures beyond yielding and followed by unloading, which results in certain residual shapes and stresses as shown in Fig. 1. The lateral surface of the bent specimens was imaged using the scanning electron microscopy (SEM).

High-energy synchrotron $\mathrm{x}$-ray diffraction measurements of local strain fields of elastically and plastically bent BMGs were performed at beamline 11-ID-C of the Advanced Photon Source (APS), Argonne National Laboratory. The energy of the incident monochromatic 
x-rays was $115 \mathrm{keV}$ and a beam size of $100 \mu \mathrm{m} \times 100 \mu \mathrm{m}$ was used for residual-stress mapping. As illustrated in Fig. 2(a), a bent BMG thin plate was positioned vertically in a way allowing incident $\mathrm{x}$-rays to penetrate the specimen through the width direction (i.e., perpendicular to the $x y$ plane). As such, we define that the $y$ direction points from the outer side surface to the inner side surface of the bent plate (i.e., the transverse direction), while the $x$ direction points downward (i.e., the longitudinal direction), as also shown in Fig. 1. By translating the BMG plate along $y$ with a step of $50 \mu \mathrm{m}$, one has a scan line across from the outer side to the inner side. A total of 5 scan lines were measured for each of the elastically and plastically bent BMGs. For each location on a scan line, diffracted x-rays were collected for 30 seconds using a PerkinElmer area detector, which was placed at $\sim 31.5 \mathrm{~cm}$ behind the specimen. Figures 2(b)-2(d) show three typical two-dimensional diffraction ring patterns recorded on the detector with respect to the polar coordinates $(q, \theta)$, where $q$ is the magnitude of momentum transfer and $\theta$ is the angle as defined in Fig. 2. These three figures correspond to a stress-free BMG, an elastically bent BMG and a plastically bent BMG, respectively.

Diffraction patterns were extracted by azimuthally averaging the ring pattern over an arc of $\pm 5^{\circ}$ with respect to a series of $\theta_{i}$ values using the Fit2D program [17],

$$
I_{i}(q)=\int_{\theta_{i}-\pi / 36}^{\theta_{i}+\pi / 36} I(q, \vartheta) d \vartheta
$$

where $\theta_{i}=\frac{\pi}{18}(i-1)$ and $i=1,2 \ldots 36 . I_{i}(q)$ was then corrected by background subtraction, polarization, fluorescence, absorption and Compton scattering to obtain the structure factor $S_{i}(q)$, as shown in Fig. 3(a), using the PDFgetX2 program [18]. For each $S_{i}(q)$, one can determine the directional strain $\left(\varepsilon_{i}\right)$ from the relative shift in position of the first diffraction peak $\left(q_{1}^{i}\right)$ with respect to $\left(q_{1}^{0}\right)$ of the stress-free BMG $[19,20]$, i.e., $\varepsilon_{i}=q_{1}^{0} / q_{1}^{i}-1$, as shown in the 
inset of Fig. 3(a). According to Poulsen et al. [21], the strain tensor $\left(\varepsilon_{i j}\right)$ can be determined by fitting the angular variation of $\varepsilon_{i}$ to the following expression

$$
\varepsilon_{i}=\varepsilon_{x x} \cos ^{2}\left(\theta_{i}\right)+\gamma_{x y} \cos \left(\theta_{i}\right) \sin \left(\theta_{i}\right)+\varepsilon_{y y} \sin ^{2}\left(\theta_{i}\right),
$$

where $\varepsilon_{x x}, \varepsilon_{y y}$ and $\gamma_{x y}\left(=2 \varepsilon_{x y}\right)$ represent the longitudinal strain, the transverse strain and the inplane engineering shear strain, respectively.

\section{Results}

Figure 3(b) shows the periodic angular variation of $\varepsilon_{i}$ (with respect to $\theta_{i}$ ) for a series of measurement locations (stepwise-changed y-values) from the outer side to the inner side along the central scan line of the elastically bent BMG. For each location, $\varepsilon_{i}$ reaches either maximum or minimum at angles of $\pi / 2, \pi, 3 \pi / 2$ and $2 \pi$ for all measurement locations, indicative of uniaxial tension or compression along $x$ where the principle strain axes coincide with the $x$ and $y$ directions. This can also be seen from the diffraction ring pattern in Fig. 2(c), which is elliptical with the major and minor axes lying along the $x$ and $y$ directions. In contrast, the angular variation of $\varepsilon_{i}$ for the plastically bent BMG is distinctly different; $\varepsilon_{i}$ reaches either maximum or minimum at angles away from $\pi / 2, \pi, 3 \pi / 2$ and $2 \pi$, which vary significantly for different locations. This is consistent with the diffraction ring pattern in Fig. 2(d), which appears to be elliptical with the major and minor axes being inclined to the $x$ and $y$ directions. The in-plane shear strain $\left(\gamma_{x y}\right)$, is thus expected to be significant, as will be discussed later.

Results for the five scan lines on the elastically bent sample in Fig. 4(a) are given in Fig. 4(b) with "+" and "-" denoting tension and compression, respectively. The longitudinal strain varies linearly from the maximum tensile strain to zero and then to maximum compressive strain from the outer to inner side of the bent beam, and the trend is reversed for the transverse strain. 
No shear strain $\gamma_{x y}$ is found for such a pure bending case. It is straightforward to find Poisson's ratio which is equal to the negative ratio of the transverse to longitudinal strains, being 0.365 that is consistent with the value from ultrasonic measurement (being 0.370 in [9]).

Along the five scan lines in the plastically bent sample, $\varepsilon_{x x}$ varies differently as shown in Fig. 4(d). Because of three-point bending, lines 1, 4, and 5 were subjected to very small bending moment, and thus nearly zero strains were found at the unloading state. The middle loading pin is located somewhere between scan line 2 and scan line 3. Along these two scan lines, $\varepsilon_{x x}$ is slightly compressive on the tensile side and changes to tensile when traversing from the outer to the inner side of the bent sample. The residual tensile strain reaches maximum at the border between shear-banding zone and non-shear-banding zone. And then this residual strain gradually varies from the maximum tension to maximum compression at the other elastic-plastic boundary. Near the inner surface, it transitions to a slightly tensile state. The corresponding strain component $\varepsilon_{y y}$ has an opposite variation as shown in Fig. 5(a).

Our previous stress analysis [12] suggests that during bending, the stress state will reach yield at the outer and inner sides of the sample in the $y$ direction. Upon unloading, it is equivalent to superpose an elastic bending stress field with a negative bending moment that balances the loading moment. This previous work also considers the yield stress asymmetry of BMG, i.e., the yield stress in compression is larger than that in tension. Note that the plastically bent samples here and in [12] are the same ones. This earlier residual stress analysis of the plastically bent sample gives the predicted dashed line in Fig. 5(a), which has identical trend as that of the measured residual strain. Our direct measurements in this paper hereby validate the analytic model in Wang et al. [12], which however did not directly measure the residual strain/stress fields. Alternatively, they found that compressive residual stress slightly improves hardness, 
while tensile residual stress dramatically decreases hardness as shown by previous finite element simulations $[12,22]$. Correspondingly, the residual compressive strain on the outer side gives rise to the slightly larger hardness when compared to the reference undeformed sample, while the tensile residual strain contributes to the reduced hardness on the inner side. Thus the hardness measurements provide an indirect spatial mapping of residual stress.

In our direct strain measurements by synchrotron x-ray diffraction, it has been found that shear strains in the elastically and plastically bent samples are distinct. Shear strain in the elastically bent sample is almost zero, which clearly results from the pure bending stress state since the bent sample is constrained in a ring in Fig. 1. On plastically bent samples, significant shear strain components have been observed. As shown in Fig. 5(c), $\gamma_{x y}$ along the scan line 2 is slightly negative and changes gradually to zero at the elastic-plastic deformation boundary where residual strain $\varepsilon_{x x}$ reaches maximum. Then it reaches the maximum near the center line of the sample where residual strain $\varepsilon_{x x}$ is nearly zero. A reversal is found when moving towards the inner surface side. These shear strains were not revealed by the indirect strain measurement method in [12], because the indentation hardness will change significantly when the stress tensor in the plane that is normal to the indentation direction has a large tensile principal stress. In other words, other residual stress/strain components cannot be determined.

The development of local shear strain fields is a result of the three-point bending in plastic deformation. On the two sides of the central loading pin, regardless of the distance from this loading pin, opposite shear stresses will be developed with a magnitude being the ratio of the applied force to the cross sectional area. As shown in Fig. 5(b), during elastic loading, $\sigma_{x y}$ on the marked five positions are roughly the same, but $\sigma_{x x}$ varies from tensile to compressive when traversing from "A" to "E". At the maximum applied load, "A" deforms plastically in a stress 
state of tensile longitudinal stress and shear stress, and "E" plastically with compressive longitudinal stress and shear stress. The unloading process is equivalent to superposing an elastic bending (which is again a three-point bending, not a pure bending). When superposing this elastic stress field, it has been found that the longitudinal stress in " $\mathrm{A}$ " becomes negative, so that the proportional elastic unloading ensures the shear stress becomes negative as well. The trend in "E" will be similar. In the elastically deformed zone near "C", the unloading does not change the signs of all the stress components, so the longitudinal stress field resembles the elastic bending stress field and the shear stress still remains positive. Since the scan line 2 is located on the other side of the central loading pin, the shear stress distribution is opposite to that of the scan line 3.

\section{Conclusion}

In summary, the local strain fields in BMG thin plates under both elastic and plastic bending were investigated by in situ high energy X-ray scattering. Under the elastic bending, strain fields vary linearly from the outer surface to inner surface of the sample. Plastically bent BMGs have much more complicated residual strain variations. While confirming a previously proposed indirect spatial measurement of residual stresses [12], the present work can determine all the strain components. Noticeably, shear strains were found in the plastically bent BMGs, which results from the three-point bending that introduces shear during loading. The capability demonstrated in this work can be utilized to probe internal strain fields in BMGs or BMG composites which can then be used to understand key processes that control shear banding behavior in these materials.

\section{Acknowledgements}


This work was sponsored by the US Department of Energy, Basic Energy Sciences, Materials Sciences and Engineering Division. Use of the Advanced Photon Source (APS), an Office of Science User Facility operated for the U.S. Department of Energy (DOE) Office of Science by Argonne National Laboratory, was supported by the U.S. DOE under Contract No. DE-AC02-06CH11357. ADS and DM's work on synchrotron x-ray data analysis was supported by the Scientific User Facilities Division, Office of Basic Energy Sciences, U.S. Department of Energy. 


\section{References}

[1] Johnson WL. MRS Bulletin 1999;24:42.

[2] Inoue A. Acta Mater 2000;48:279.

[3] Chen MW. Annu Rev Mater Res 2008;38:14.

[4] Cheng YQ, Ma E. Prog Mater Sci 2011;56:379.

[5] Bei H, Xie S, George EP. Phys Rev Lett 2006;96:105503.

[6] Hofmann DC, Suh JY, Wiest A, Duan G, Lind ML, Demetriou MD, Johnson WL. Nature 2008;451:1085.

[7] Wu Y, Xiao YH, Chen GL, Liu CT, Lu ZP. Adv Mater 2010;22:2770.

[8] Pauly S, Gorantla S, Wang G, Kuhn U, Eckert J. Nat Mater 2010;9:473.

[9] Li WD, Bei H, Tong Y, Dmowski W, Gao YF. Appl Phys Lett 2013;103:171910.

[10] Zhang Y, Wang WH, Greer AL. Nat Mater 2006;5:857.

[11] Chen LY, Ge Q, Qu S, Jiang QK, Nie XP, Jiang JZ. Appl Phys Lett 2008;92:211905.

[12] Wang L, Bei H, Gao YF, Lu ZP, Nieh TG. Acta Mater 2011;59:2858.

[13] Wang L, Bei H, Gao YF, Lu ZP, Nieh TG. Acta Mater 2011;59:7627.

[14] Jia HL, Liu FX, An ZN, Li WD, Wang GY, Chu JP, Jang JSC, Gao YF, Liaw PK. Thin Solid Films 2014;561:2.

[15] Narayan RL, Singh PS, Hofmann DC, Hutchinson N, Flores KM, Ramamurty U. Acta Mater 2012;60:5089.

[16] Jia HL, Zheng LL, Li WD, Li N, Qiao JW, Wang GY, Ren Y, Liaw PK, Gao YF. Metall Mater Trans A 2015;46:2431.

[17] Hammersley AP. FIT2D: An Introduction and Overview, ESRF Internal Report, ESRF97HA02T (1997).

[18] Qiu X, Thompson JW, Billinge SJL. J Appl Cryst 2004;37: 678.

[19] Hufnagel TC, Ott RT, Almer J. Phys Rev B 2006;73:064204.

[20] Ma D, Stoica AD, Wang XL, Lu ZP, Clausen B, Brown DW. Phys Rev Lett 2012;108:085501.

[21] Poulsen HF, Wert JA, Neuefeind J, Honkimaki V, Daymond M. Nat Mater 2005;4:33.

[22] Chen LY, Ge Q, Qu S, Jiang JZ. Scripta Mater 2008;59:1210. 


\section{Figure Captions}

FIG. 1: $\quad$ Schematic illustration of the elastically bent BMG sample (which is constrained in a steel ring) in (a) and the plastically bent one by three point bending in (b). Five scan lines mapped by high-energy x-ray beams are marked in the bent samples in (c).

FIG. 2: (a) Coordinates and symbols defined in the strain analysis. (b)-(d) Representative diffraction images without applied strain in (b), with principal strains lying in $x$ and $y$ directions in (c), and with principle strains lying along the two dashed lines in (d). The angle $\theta$ is used for tensor transformation in Eq. (2).

FIG. 3: (a) The structure factor $S_{i}(q)$ with respect to the longitudinal direction $\left(\theta_{i}=\pi / 2\right)$ for a stress-free BMG and an elastically bent BMG, with the inset showing the shift in position of the first diffraction peak. The directional strain $\varepsilon_{i}$, as calculated from the relative peak shift, varies against $\theta_{\mathrm{i}}$ and scanning distance $y$ (along the central scan line) in the (b) elastically and (c) plastically bent samples.

FIG. 4: $\quad$ SEM images of the elastically and plastically bent samples with the five scan lines in (a) and (c), respectively. Three strain components are plotted for the elastically bent sample in (b), while only $\varepsilon_{x x}$ for plastically bent sample in (d).

FIG. 5: (a) Comparisons between the directly measured strain fields and the analytical residual stress analysis in Wang et al. [12] along the scan line 3. (b) The corresponding plastically bent sample and representative locations. (c) $\gamma_{x y}$ on these two scan lines are opposite to each other. 


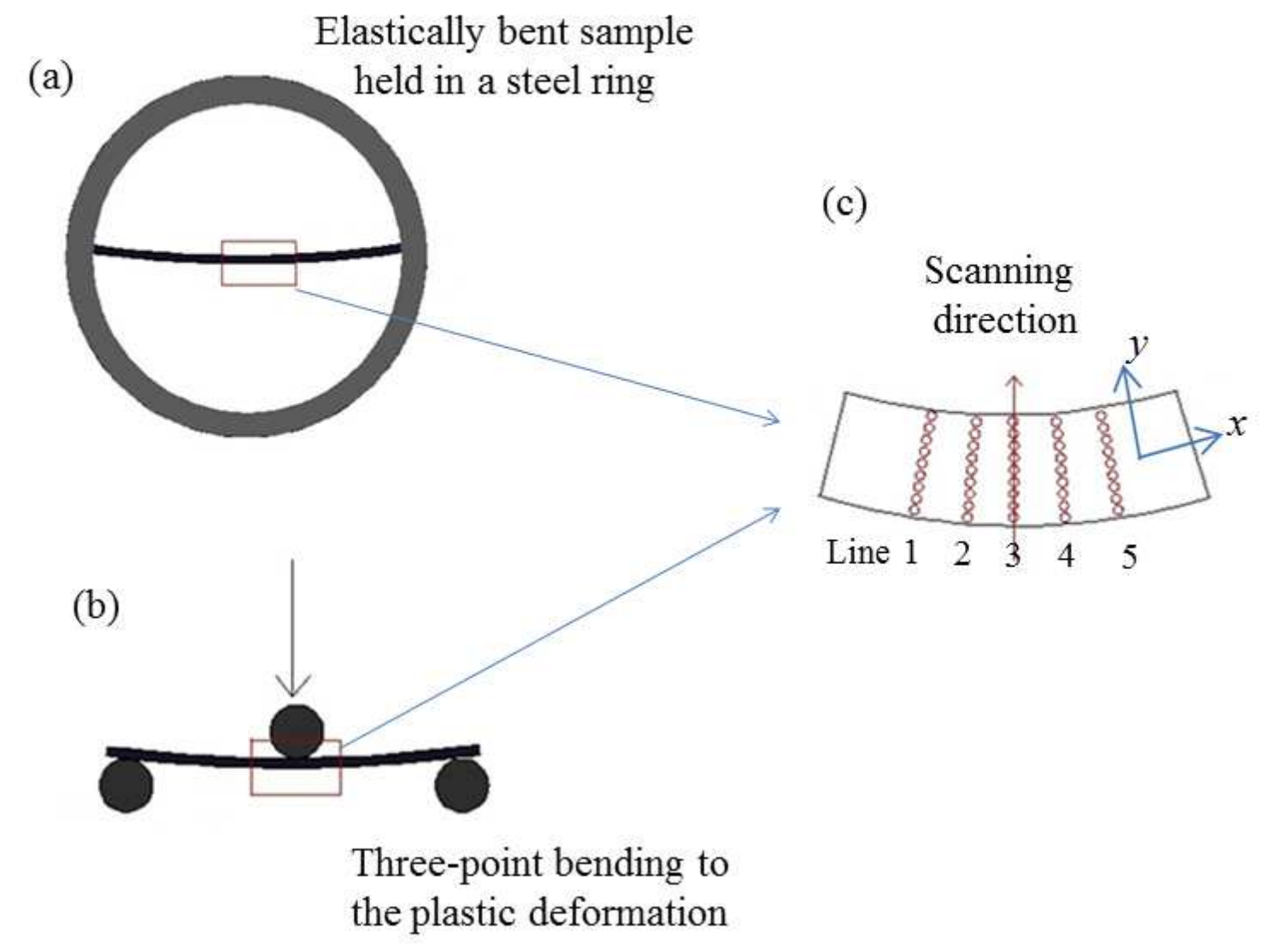

Figure 1

1

3

4

5

7

8
9

10

11

12

13

16

17

18

19

20

22

23

26

27

28

30

31

32

33

35

36

37

38

39

40

41

42

43

44

48

49

50

51

53

54

55

56

58

59

60

61

62

63

65 

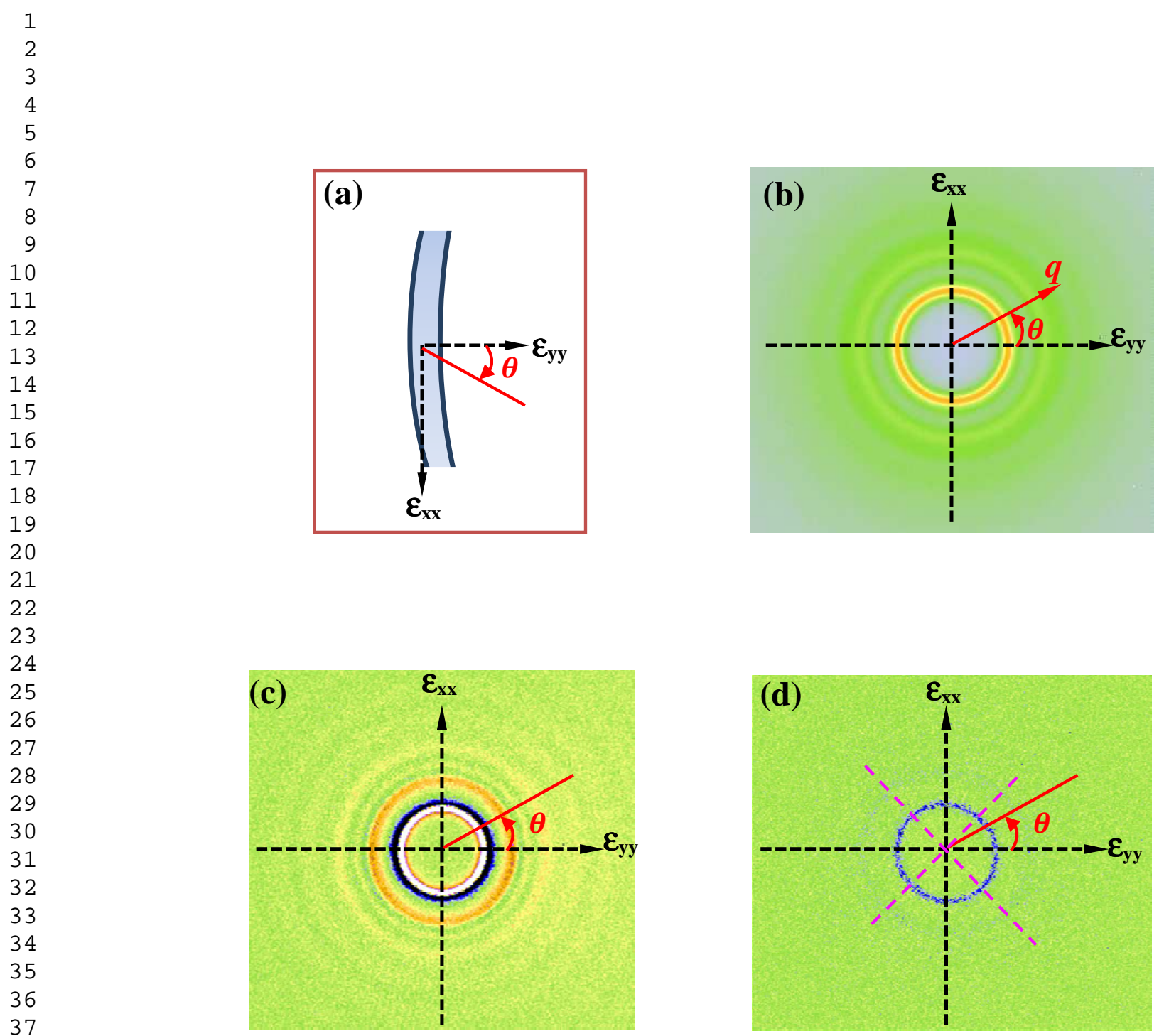
(a)

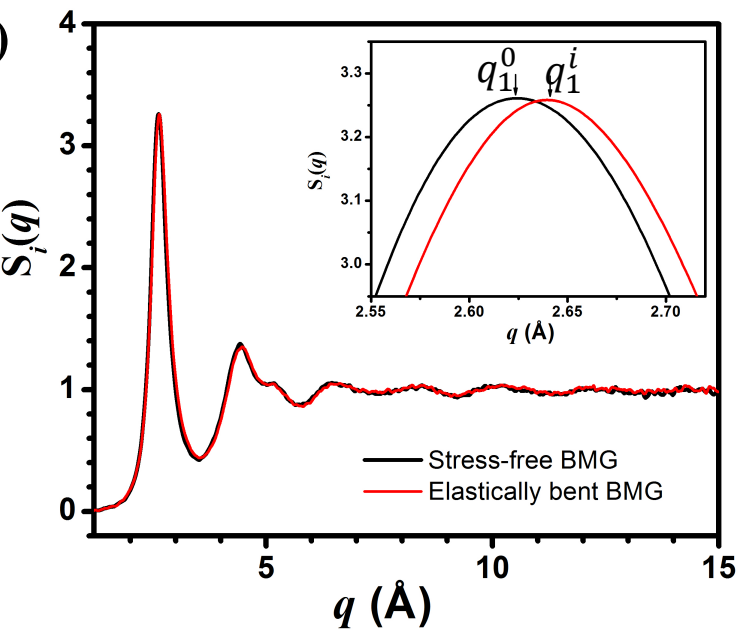

(b)

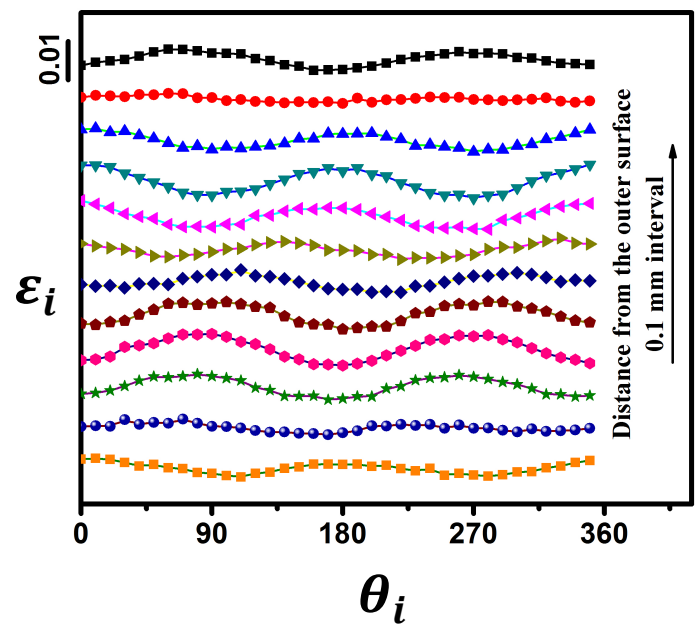

(c)

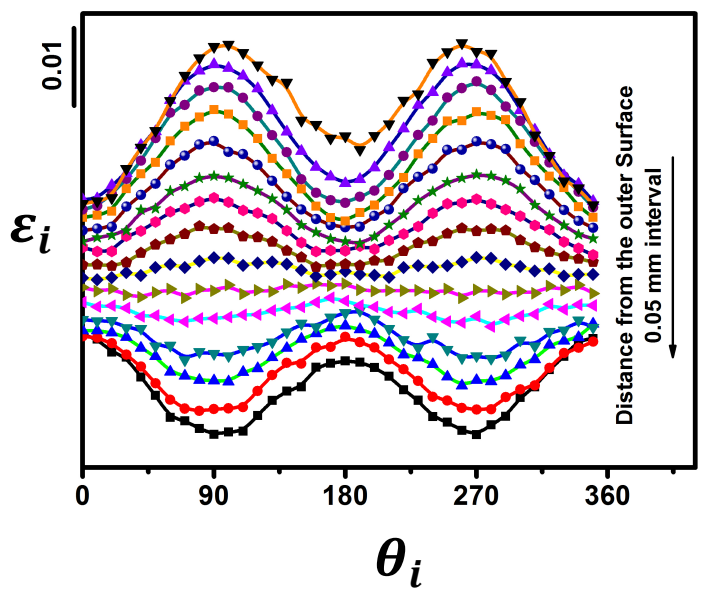

Figure 3 

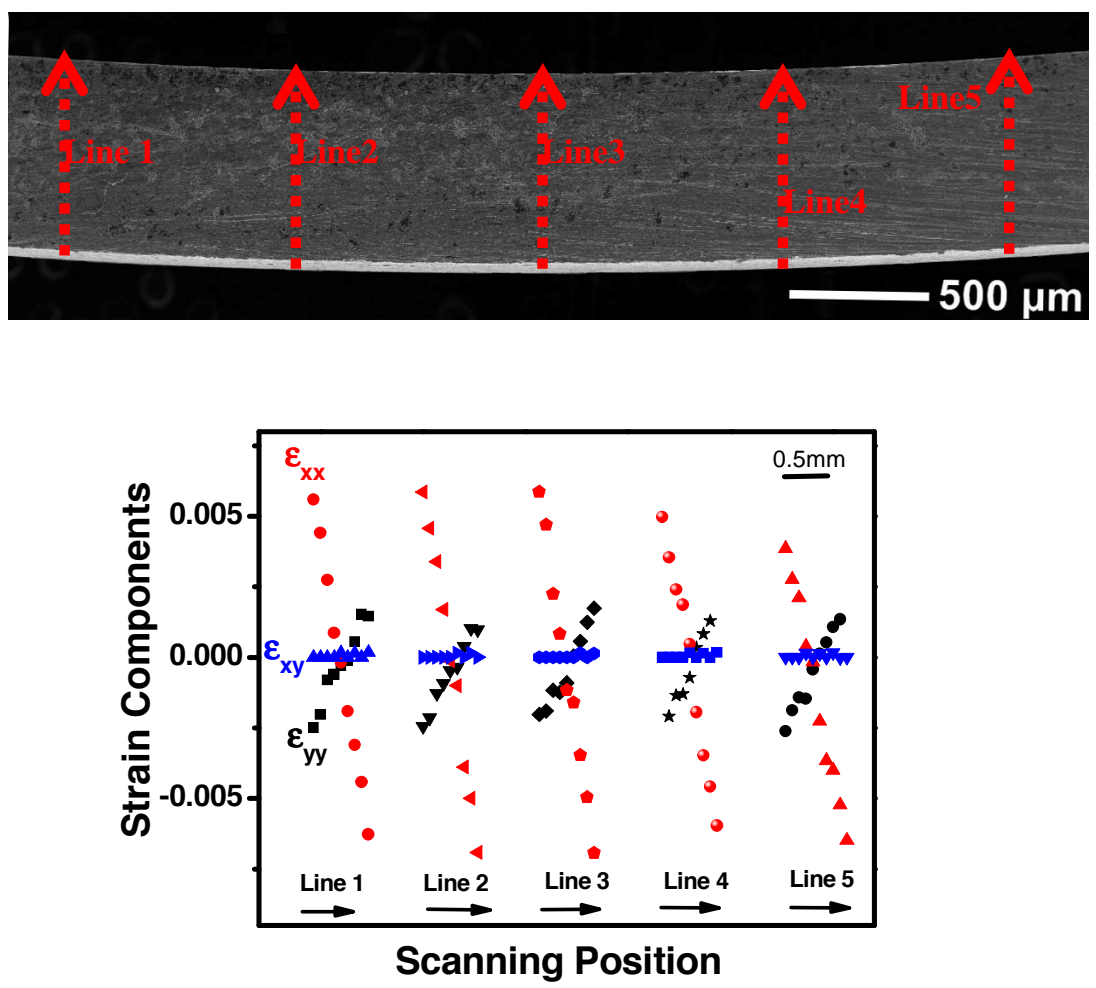

(b)

(c)
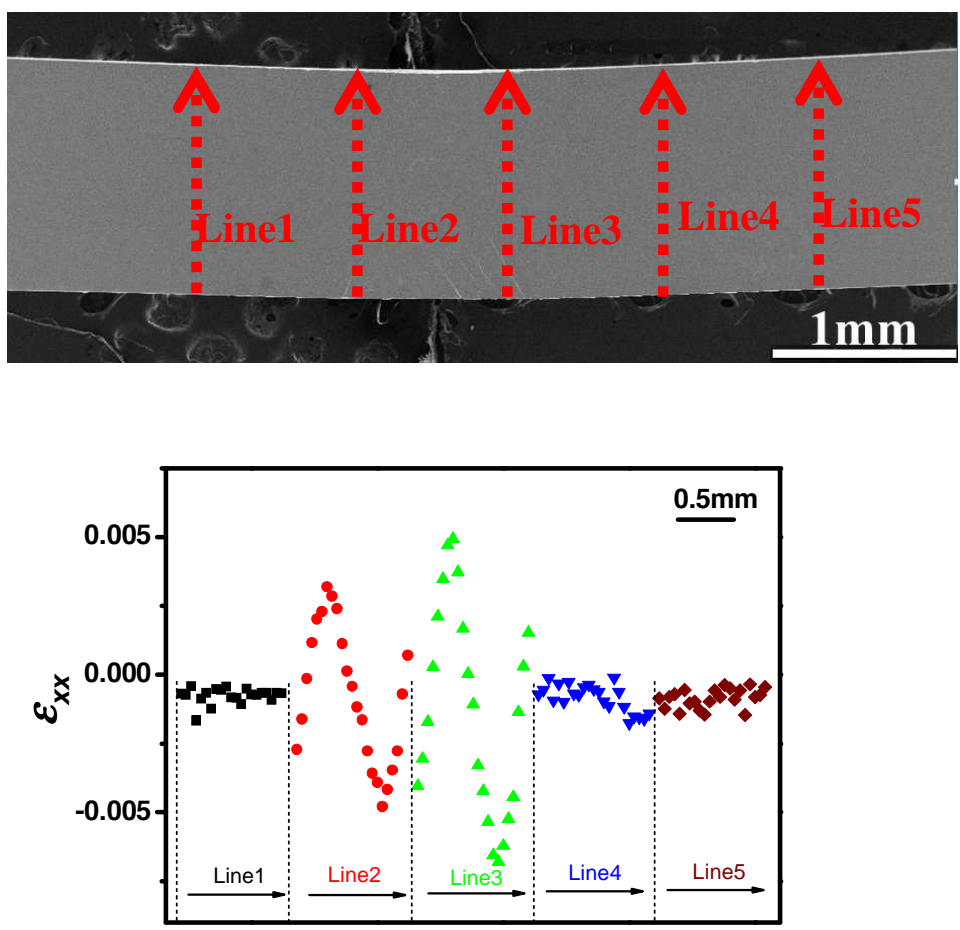

Scanning Position

(d)

Figure 4 

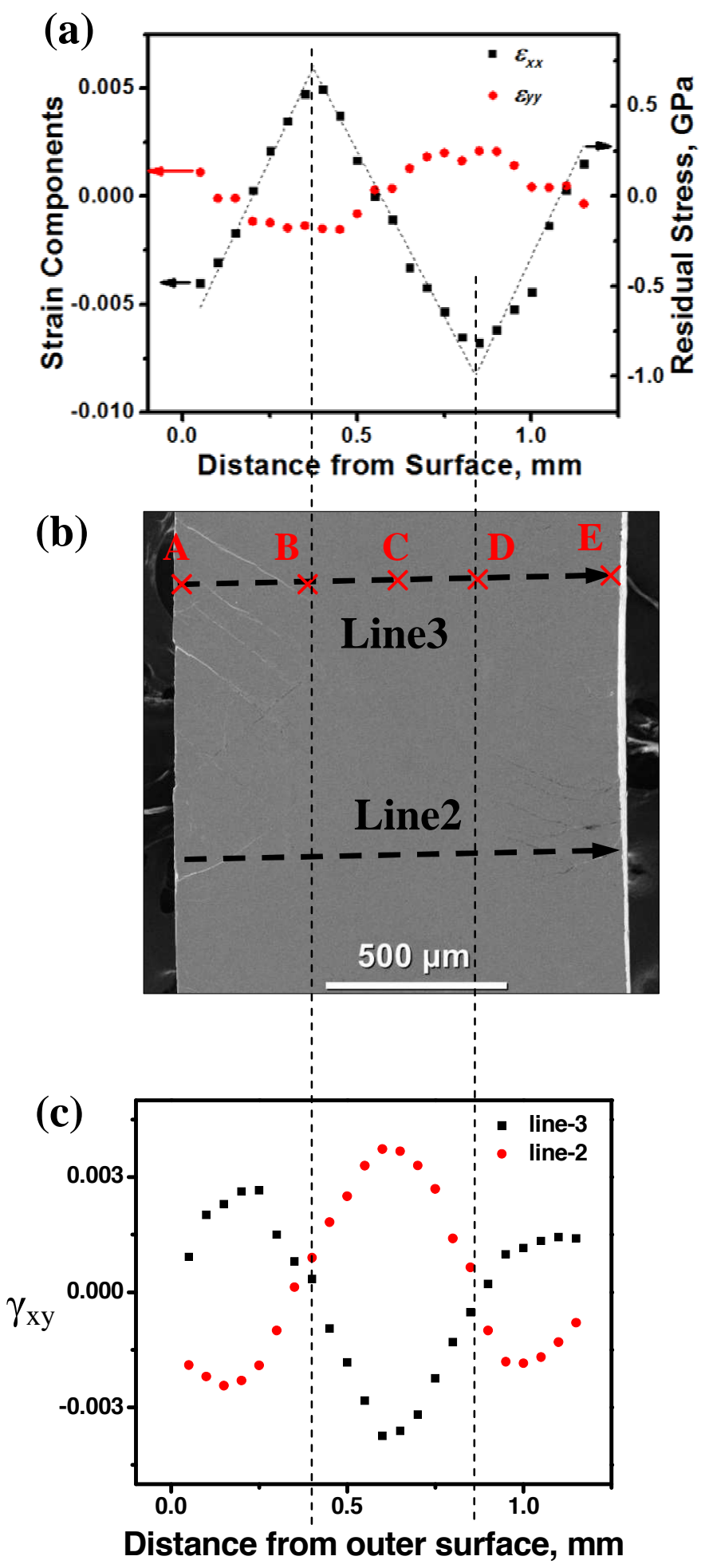

Figure 5 

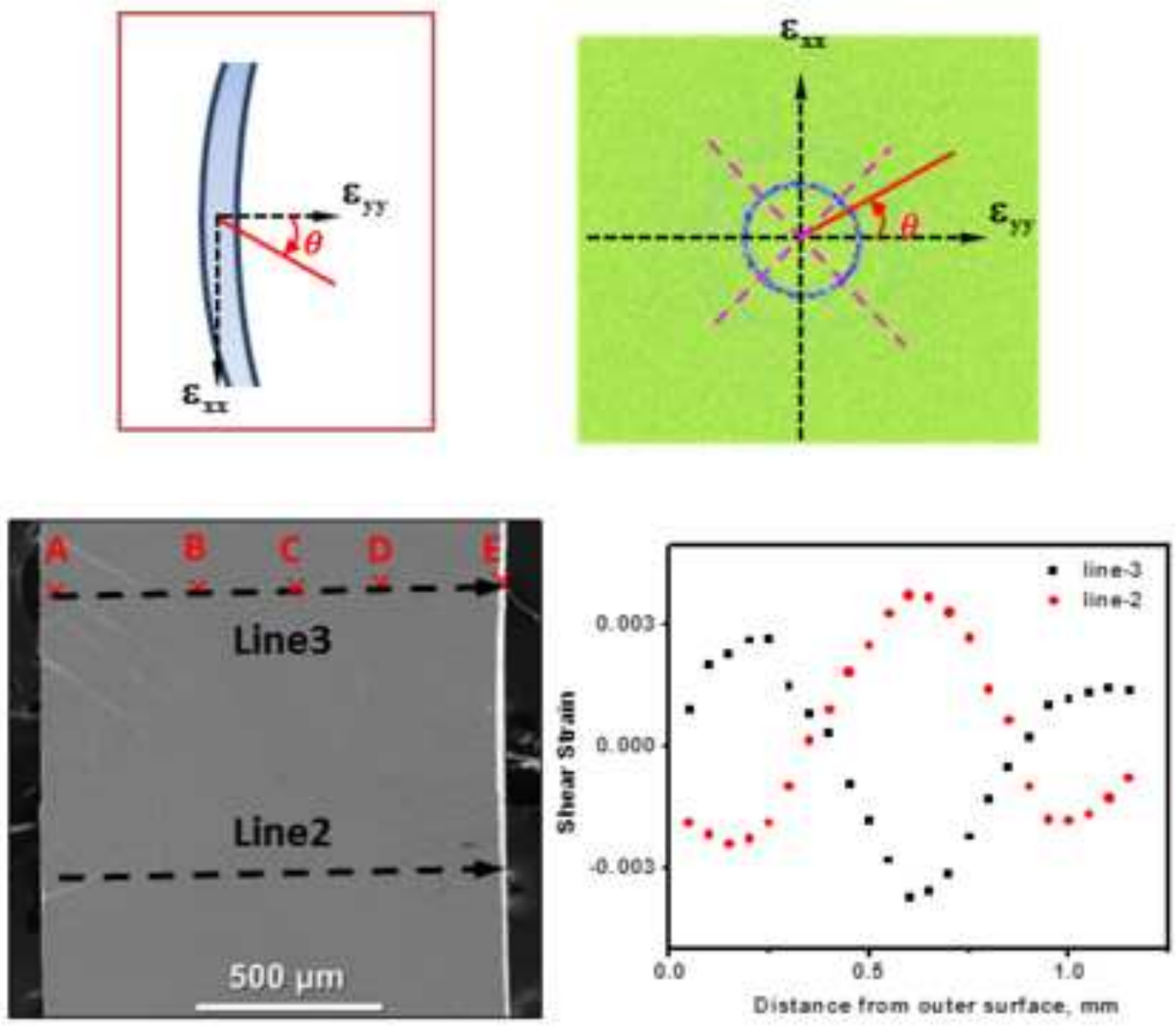